Research Article

\title{
Higher Order of Convergence with Multivalued Contraction Mappings
}

\author{
Jia-Bao Liu $\mathbb{D}^{1},{ }^{1}$ Asma Rashid Butt, ${ }^{2}$ Shahzad Nadeem, ${ }^{3}$ Shahbaz Ali ${ }^{1},{ }^{4}$ \\ and Muhammad Shoaib ${ }^{5}$ \\ ${ }^{1}$ School of Mathematics and Physics, Anhui Jianzhu University, Hefei 230601, China \\ ${ }^{2}$ Department of Mathematics, University of Engineering and Technology, Lahore, Pakistan \\ ${ }^{3}$ Department of Mathematics, Quaid-i-Azam University, Islamabad-45320, Pakistan \\ ${ }^{4}$ Department of Mathematics, Khawaja Fareed University of Engineering and Information Technology, \\ Rahim Yar Kahn, Pakistan \\ ${ }^{5}$ Department of Mathematics, University of Punjab, New Campus, Lahore, Pakistan
}

Correspondence should be addressed to Shahbaz Ali; shahbaz.ali@kfueit.edu.pk

Received 14 September 2020; Revised 30 October 2020; Accepted 2 November 2020; Published 11 November 2020

Academic Editor: Xiaolong Qin

Copyright (c) 2020 Jia-Bao Liu et al. This is an open access article distributed under the Creative Commons Attribution License, which permits unrestricted use, distribution, and reproduction in any medium, provided the original work is properly cited.

In this paper, we establish some theorems of fixed point on multivalued mappings satisfying contraction mapping by using gauge function. Furthermore, we use $Q$ - and $R$-order of convergence. Our main results extend many previous existing results in the literature. Consequently, to substantiate the validity of proposed method, we give its application in integral inclusion.

\section{Introduction}

A well-known mathematician, Banach, gave a main source of the existence of fixed points. An iterative method was used in the Banach contraction principle which converges to the fixed point linearly. In order to obtain higher order of convergence, Proinov [1] generalized Banach contraction theorem by generalizing the contractive condition which involves a gauge function of order $r \geq 1$. Later, Kiran and Kamran [2] extended his work and generalized using multivalued maps from a complete metric space into the space of all nonempty proximinal closed spaces. In this context, we study the multivalued contraction mapping involved in metric space from [3-7]. And some details for multivalued mapping and their fixed points are included in [1, 2, 8-15]. Recently, Petrusel investigated the local fixed point results for graphic contractions and multivalued locally contractive operators and proposed their application in optimization theory in $[16,17]$. Sow proposed the strong convergence of a modified Mann algorithm for multivalued quasi-nonexpansive mappings and monotone mappings with an application in $[18,19]$. (Proinov, General local convergence theory for a class of iterative processes and its applications to
Newton's method. Journal of Complexity, 25, 1, 38-62). We extend some results of Proinov to the case of multivalued maps from a metric space $V$ into the space of all bounded closed subspace of $V$. We recall some notations which we used in this paper. Let $(V, d)$ be a metric space and $A$ be a subset of $V$; distance from every $v \in V$ to subset $A$ is defined as there is a element $a \in A$ such that $d(v, a)=d(v, A)$. Now, distance from the point to set is defined as $v \in V$ and $A \subset V$, where $d(v, A)=\inf \{d(v, w): w \in A\}$. We denote $\mathrm{CB}(V)$ as the class of all nonempty closed and bounded subsets of $V$. Let $H$ be the generalized Hausdorff metric on $\mathrm{CB}(V)$ generated by metric $d$ as

$$
H(A, B)=\max \left\{\sup _{v \in A} d(v, B), \sup _{w \in B} d(w, A)\right\},
$$

for each $A, B \in \mathrm{CB}(V)$. The fixed point of $T: V \longrightarrow \mathrm{CB}(V)$ is a point $v \in V$ if $v \in T v$. Throughout this paper, $L$ denotes an interval on $\mathbb{R}_{+}$containing 0 , this interval is represented as $[0, A] t, n[0, a)$ or $[0, \infty)$, and for polynomial, we used $S_{b}(e)=1+e+\cdots+e^{b-1}$. We use the order of convergence as $Q$-order and $R$-order with $r \geq 1$ for iterative process: 


$$
v_{b+1} \in T v_{b}, \quad b=0,1,2, \ldots
$$

and use gauge function $\varrho$ on $L$, that is, $\varrho: L \longrightarrow L$. In this paper, we present some theorems of fixed point on multivalued mappings satisfying contraction mapping by using gauge function. Also, we use $Q$ - and $R$-order of convergence. In the last section, we applied our proposed method in integral theory.

\section{Preliminaries}

In this section, we take some results and definitions from $[1,19]$.

Definition 1. A gauge mapping with order $r$ which is greater than 1 defines with $\varrho: L \longrightarrow L$ on $L$ if it fulfills the following conditions:

(1) $\varrho(\gamma e) \leq \gamma^{r} \varrho(e)$ for each $\gamma \in(0,1)$ and $e \in L$

(2) $\varrho(e) \leq e$ for each $e \in L$

The first condition of the above definition can easily show that it is equivalent to the condition $\varrho(0)=0$ and $\varrho(e) / e^{r}$ is nondecreasing on $L-0$. Fixed point theory in metric space is full of fixed point theorems in different classes of contraction, which can be obtained by different properties of gauge function $\varrho$.

Example 1. $\varrho: L \longrightarrow L$ where $L=[0, \infty)$ and $\varrho(e)=\gamma e$ where $\gamma \in(0,1)$. Then,

$$
\begin{aligned}
& \text { (1) } \varrho(\gamma e)=\gamma^{2} e=\gamma \cdot \gamma e=\gamma(\gamma e)=\gamma \varrho(e), \\
& e \in L \Rightarrow \varrho(\gamma e)=\gamma \varrho(e) \\
& \text { (2) } \varrho(e)=\gamma e<e \text { because } \varrho \in(0,1)
\end{aligned}
$$

Lemma 1. A mapping $\varrho$ of order $r$ on L fulfills the gauge condition and there is another mapping $\pi$ on $L$ which is nondecreasing and nonnegative such that

$$
\varrho(e)=e \pi(e), \quad \text { for each } e \in L \text {. }
$$

Now, the properties which the mapping $\pi$ has are

$$
0 \leq \pi(e) \leq 1, \quad \text { for each } e \in L,
$$$$
\pi(\gamma t) \leq \gamma^{r-1} \pi(t), \quad \text { for each } \gamma \in(0,1), \quad \text { and each } e \in L
$$
as

Now, the gauge mapping which is defined on $\varrho$ is written

$$
0 \leq \pi(e) \leq 1, \quad \text { for each } e \in L
$$

For Q-order of convergence with at least order $r \geq 1$, sequence $\left(v_{b}\right)$ converges to $\eta$. If a constant $c$ exists which is greater then zero such that $d\left(v_{b+1}, \eta\right) \leq c\left(d\left(v_{b}, \eta\right)\right)^{r}$ for sufficiently large $b$, then there are the different cases which depend on $r$; but, in that case, $r=1$ and $b \leq 1$. Now, the type of convergence linearly and quadratically depends on the order $r$, respectively, 1 and 2 .

Assume a sequence $\left(v_{n}\right)$ whose converges with order $R$ with constant $\eta$ and $r \geq 1$. Let us take a real number sequence $\left(\alpha_{b}\right)$ whose order of convergence is $Q$ and that converge to zero with $r \geq 1$ such that $d\left(v_{b}, \eta\right) \leq \alpha_{b}$.

\section{Main Result}

Definition 2. Let $(V, d)$ be a metric space and define a mapping $T: V \longrightarrow \mathrm{CB}(V)$ such that $H(T v, T w)$ be a Hausdorff metric defined on $\mathrm{CB}(V)$; then,

$$
\begin{gathered}
d(T v, w) \leq H(T v, T w), \\
H(T v, T w) \leq \varrho(d(v, w)),
\end{gathered}
$$

where the gauge mapping is $\varrho$.

Lemma 2. Let $V$ be a nonempty and arbitrary set and define a mapping $T: C \subset V \longrightarrow C B(V)$ such that a mapping which has the starting condition of $T$ with gauge mapping of order $r$ is @ on the interval $L$ which is $D: C \longrightarrow \mathbb{R}^{+}$. Now, for $T$, each starting point $v_{0} \in C$ and each $b \geq 0$. We have

$$
\begin{aligned}
D\left(v_{b+1}\right) & \leq \varrho\left(D\left(v_{b}\right)\right), \\
D\left(v_{b}\right) & \leq D\left(v_{0}\right) \lambda^{S_{b}(r)} .
\end{aligned}
$$

Proof. Here, $v_{b+1} \in D$ and $v_{0}$ is the starting point of $T$. As $v_{b+1} \in T v_{b}$, using Definition 2 and Lemma 2, we obtain

$$
\begin{aligned}
D\left(v_{b+1}\right) & =d\left(v_{b+1}, T v_{b+1}\right) \\
& \leq H\left(T v_{b}, T v_{b+1}\right) \\
& \leq \varrho\left(D\left(v_{b}\right)\right) .
\end{aligned}
$$

Thus, we obtain

$$
D\left(v_{b+1}\right) \leq \varrho\left(D\left(v_{b}\right)\right) .
$$

Now prove

$$
D\left(v_{b}\right) \leq E\left(v_{0}\right) \lambda^{s_{b}(r)} .
$$

As $\varrho$ is nondecreasing on $L$, we have

$$
D\left(v_{b}\right) \leq \varrho\left(D\left(v_{b-1}\right)\right) \leq \varrho\left(\varrho\left(D\left(v_{b-2}\right)\right)\right) \leq \cdots \leq \varrho^{b}\left(D\left(v_{0}\right)\right),
$$

where $\varrho$ is thw gauge function and $\lambda=\left(\varrho\left(D\left(v_{0}\right)\right)\right)^{s_{b}(r)}$. Then,

$$
\begin{aligned}
D\left(v_{b}\right) & =\varrho^{b}\left(D\left(v_{0}\right)\right) \\
& \leq D\left(v_{0}\right)\left(\varrho\left(D\left(v_{0}\right)\right)\right)^{s_{b}(r)} \\
& =D\left(v_{0}\right) \lambda^{s_{b}(r)} .
\end{aligned}
$$


Theorem 1. Assume a metric space $(V, d)$ and define a mapping $T: C \subset V \longrightarrow C B(V)$. The initial condition of $T$ is $D: C \longrightarrow \mathbb{R}_{+}$and gauge mapping $\varrho: L \longrightarrow L$ fulfills the condition $\varrho(e) \leq e$ for all $e \in L$. Let $\eta$ be in C. Assume that

$$
\begin{aligned}
H(T v, T \eta) & \leq \gamma(D(x)) d(v, \eta), \quad \forall v \in C \text { with } D(\eta) \in L, \\
d(T v, \eta) & \leq H(T v, T \eta),
\end{aligned}
$$

where $\gamma$ is a nondecreasing mapping on $L$ such that $\gamma(e) \in[0,1), \forall e \in L$. Then, $T$ has a unique fixed point $\eta$ at which we define $E=\{v \in C: D(v) \in L\}$. Furthermore, for every starting point $v_{0}$ of $T$, Picard iteration $v_{b+1} \in T v_{b}$ remains in $V$ and converges $Q$-linearly to $\eta$ with error which we can estimate as

$$
\begin{aligned}
d\left(v_{b+1}, \eta\right) & \leq g d\left(v_{n}, \eta\right), \\
d\left(v_{n}, \eta\right) & \leq g^{b} d\left(v_{0}, \eta\right),
\end{aligned}
$$

where $g=\gamma D\left(v_{0}\right)$.

Proof. Substitute $v=\eta$ and obtain

$$
d(T \eta, \eta) \leq H(T \eta, T \eta) \leq \gamma D(\eta) d(\eta, \eta),
$$

where $d(\eta, \eta)=0$.

Thus,

$$
d(T \eta, \eta)=0, \quad \text { this implies that } \eta \in T \eta .
$$

Now, we prove that $\eta$ is unique. Suppose that contrary $\mu$ is another fixed point such that $\mu \in T \mu$ and $\mu \neq \eta$. Then,

$$
\begin{aligned}
d(\eta, \mu) & \leq H(T \eta, T \mu) \\
& \leq \gamma(D(\eta)) d(\eta, \mu) \\
& <d(\eta, \mu) .
\end{aligned}
$$

This implies that

$$
d(\eta, \mu)<d(\eta, \mu),
$$

which is a contradiction such that

$$
\eta=\mu,
$$

given $v_{0}$ is the initial point of Picard iteration. We obtain

$$
d\left(v_{b+1}, \eta\right) \leq H\left(T_{v_{b}}, T \eta\right) \leq \gamma\left(D\left(v_{b}\right)\right) d\left(v_{b}, \eta\right),
$$

as $D\left(v_{b}\right) \leq D\left(v_{0}\right)$. Then, we obtain $\gamma\left(E\left(v_{b}\right)\right) \leq \gamma\left(E\left(v_{0}\right)\right)$. Now, following from the above inequality, we obtain

$$
d\left(v_{b+1}, \eta\right) \leq H\left(T_{v_{b}}, T \eta\right) \leq \gamma\left(D\left(v_{b}\right)\right) d\left(v_{b}, \eta\right),
$$

and this implies that

$$
\begin{aligned}
& d\left(v_{b+1}, \eta\right) \leq \gamma\left(D\left(v_{b}\right)\right) d\left(v_{b}, \eta\right) \leq \gamma\left(D\left(v_{0}\right)\right) d\left(v_{b}, \eta\right), \\
& d\left(v_{b+1}, \eta\right) \leq \gamma\left(D\left(v_{0}\right)\right) d\left(v_{b+1}, \eta\right),
\end{aligned}
$$

given $g=\gamma\left(D\left(v_{0}\right)\right)$. So

$$
d\left(v_{b+1}, \eta\right) \leq g d\left(v_{b}, \eta\right)
$$

and $d\left(v_{b}, \eta\right) \leq g d\left(v_{b-1}, \eta\right) \leq g\left(g d\left(v_{b-2}, \eta\right)\right), \ldots, g^{b} d\left(v_{0}, \eta\right)$. Therefore,

$$
d\left(v_{b}, \eta\right) \leq g^{b} d\left(v_{0}, \eta\right) .
$$

This proof is complete and it is necessary to note that $0 \leq g \leq 1$.

Remark 1 . Let $\eta$ be a number in $C$. It is easily represented that following the assumption of theorem, the important point $D(\eta) \in L$ is fulfilled iff the gauge mapping has $\varrho$ as the fixed point. Now, take $\eta$ as the root of mapping $C$ which can be selected from the theorem. So the fixed point of $\varrho$ is 0 .

Remark 2. Without using the measurement of the error, the above result will be true. If gamma is used as one of the nondecreasing mappings on $L$, then gamma is right continuous.

Theorem 2. Assume a metric space $(V, d)$ and define a mapping $T: C \subset V \longrightarrow C B(V)$. The initial condition of $T$ is $D: C \longrightarrow \mathbb{R}_{+}$and gauge mapping $\varrho: L \longrightarrow L$ fulfills the condition $\varrho(e) \leq e$ for all $e \in L$. Let $\eta$ be in C. Assume that

$$
\begin{aligned}
H(T v, T \eta) & \leq \gamma(D(v)) d(v, \eta), \quad \forall v \in C \text { with } D(\eta) \in L, \\
(T v, \eta) & \leq H(T v, T \eta),
\end{aligned}
$$

where $\gamma$ is a nondecreasing mapping on $L$ such that e $\gamma(e)$ is a gauge mapping which is strict of order $r$ on L. Furthermore, for $e \in L: \omega(e)=0$, it implies that $\gamma(e)=0 \omega$ is a nondecreasing nonnegative function on J fulfilling

$$
\varphi(e)=e \omega(e), \quad \forall e \in L .
$$

Then, $\eta$ is fixed point of $T$ which is unique with $E=\{v \in C: D(v) \in L\}$. Furthermore, for every starting point $v_{0}$ of $T$, the following conditions are fulfilled:

(1) The iterative sequence $v_{b+1} \in T v_{n}$ is in the set $E$ and it will converge to $\eta$. If $\omega\left(D\left(v_{0}\right)\right)<1$, then the sequence $v_{b+1} \in T v_{b}$ converges with $R$-order $r$.

(2) For each $b \geq 0$, the error estimate is

$$
d\left(v_{b}, \eta\right) \leq \phi^{n} \mu^{s_{b}(r)} d\left(v_{0}, \eta\right)
$$

where $\mu=\omega\left(D\left(v_{0}\right)\right), \phi=\psi\left(D\left(v_{0}\right)\right)$, and $\psi$ is a nonnegative mapping of $L$ such that $\gamma(e)=\omega(e) \psi(e)$ for each $e \in L$.

(3) If there is another mapping which is continuous $B: G \longrightarrow \mathbb{R}_{+}$at $\eta$, then $D(v) \leq B(v) d(v, \eta), \forall v \in G$. Hence, we use the sequence which is iterative $v_{b+1}=$ $T v_{b}$ and converges to $\eta$ with the order Q- with $r$ as

$$
d\left(v_{b+1}, \eta\right) \leq B_{v}\left(d\left(v_{b}, \eta\right)\right)^{r}, \quad \forall b \geq 0,
$$


where $B_{v}=\left(B\left(v_{b}\right)\right)^{r-1} \eta\left(D\left(v_{b}\right)\right) \longrightarrow B(\eta)^{r-1} \eta(0)$ is $b \longrightarrow \infty$ and a mapping which is real, $\eta: L \longrightarrow \mathbb{R}_{+}$ is continuous at 0 , and $\gamma(e)=e^{r-1} \eta(e)$ for all $e \in L$.

Proof. We have already proved that fixed point is unique. Now, we have to prove $R$-order of convergence. Given that $\gamma(e)=$ $\omega(e) \psi(e)$ for all $e \in L$, we select a arbitrary number $b$ which is nonnegative and represented as $\tau: L \longrightarrow \mathbb{R}_{+}$as follows:

$$
\tau(e)= \begin{cases}\frac{\gamma(e)}{\omega(e)}, & \text { if } \omega(e)>0, \\ b, & \text { if } \omega(e)=0 .\end{cases}
$$

Now, by result, we observe that

$$
\begin{aligned}
& 0 \leq \mu \leq 1, \\
& \omega \geq 0,
\end{aligned}
$$

and $0 \leq \omega \mu<1$. Then,

$$
\begin{aligned}
d\left(v_{b+1}, \eta\right) & \leq H\left(T_{v_{b}}, T \eta\right) \\
& \leq \gamma\left(E\left(v_{b}\right)\right) d\left(v_{b}, \eta\right) \\
& \leq \gamma\left(E\left(v_{0}\right)\right) d\left(v_{b}, \eta\right) \\
& \leq d\left(v_{b}, \eta\right) \\
& \leq d\left(v_{b}, 0\right) .
\end{aligned}
$$

Here, $d\left(v_{b}, \eta\right)=\alpha_{b}$. According to abovementioned lemma, the function $\gamma$ satisfies

$$
\gamma(\pi e) \leq \pi^{r-1} \gamma(e), \quad \text { for all } \pi \in[0,1] \text { and } e \in L .
$$

Now, we shall prove the next part by induction. Substitute $b=0$ and obtain $d\left(v_{0}, \eta\right)=d\left(v_{0}, \eta\right)$. Assume that it fulfills for $b$. Now, we prove that for $b+1$,

$$
\begin{aligned}
d\left(v_{b+1}, \eta\right) & \leq H\left(T_{v_{b}}, T \eta\right) \\
& \leq \gamma\left(D\left(v_{b}\right)\right) d\left(v_{b}, \eta\right) \\
& \leq \gamma\left(D\left(v_{b}\right)\right) \omega^{n} \mu^{s_{b}(r)} d\left(v_{0}, \eta\right), \\
\gamma(\mu e) & \leq \mu^{r-1} \gamma(t), \quad \forall \mu \in[0,1] \text { and } e \in L, \\
\gamma\left(v_{b}\right) & \leq D\left(v_{0}\right) \mu_{b}^{s}(r), \\
\gamma\left(D\left(v_{b}\right)\right) & \leq \gamma\left(D\left(v_{0}\right)\right) \mu^{s_{b}(r)} \\
& \leq \mu^{(r-1) s_{b}(r)} \gamma\left(D\left(v_{0}\right)\right) \\
& =\omega \mu^{1+(r-1) s_{b}(r)}, \\
S_{b}(r) & =1+r+r^{2}+r^{3}+\cdots+r^{b-1}, \\
(r-1) S_{b}(r) & =r+r^{2}+\cdots+r^{b}-1-r-r^{2}-\cdots-r^{b-1} \\
& =-1+r^{n}, \\
\gamma\left(D\left(v_{b}\right)\right) & =\omega \mu^{r^{b}}, \\
d\left(v_{b+1}, \eta\right) & \leq \omega^{n+1} \mu_{b+1}^{S}(r) d\left(v_{0}, \eta\right) .
\end{aligned}
$$

Now, we have to prove that $d\left(v_{b+1}, \eta\right) \leq c_{b} d\left(v_{b}, \eta\right)^{r}, \forall b \geq 0$.

We already know that

$$
\begin{aligned}
d(T v, \eta) \leq H(T v, T \eta) & \leq \gamma(D(v)) d(v, \eta), \\
\gamma(e) & =e^{r-1} \mu(e), \\
D(v) & \leq C(v) d(v, \eta), \\
d\left(v_{b+1}, \eta\right) & \leq H\left(T_{v_{b}}, T \eta\right) \leq \gamma\left(D\left(v_{b}\right)\right) d\left(v_{b}, \eta\right) \\
& =D\left(v_{b}\right)^{r-1} \mu\left(D\left(v_{b}\right)\right) d\left(v_{b}, \eta\right) \\
& \leq C\left(v_{b}\right)^{r-1} d\left(v_{b}, \eta\right)^{r-1} \mu\left(D\left(v_{b}\right)\right) d\left(v_{b}, \eta\right) \\
& =C\left(v_{b}\right)^{r-1} \\
& =C_{b} d\left(v_{b}, \eta\right)^{r} .
\end{aligned}
$$

Corollary 1. Let $(V, d)$ be a metric space and define an operator $T: C \subset V \longrightarrow C B(V)$ and $\eta \in C$. Assume that

$$
H(T v, \eta) \leq \varrho(d(v, \eta)), \quad \forall v \in C \text { and } d(v, \eta) \in L,
$$

where a gauge mapping which is strict with order $r \geq 1$ is $\varrho$. Then, the fixed point $T$ which is unique is $\eta$ contains the set $E=\{v \in C: d(v, \eta) \in L\}$. Furthermore, if $T: V \longrightarrow V$, then for every point $v_{0} \in L$, the following conditions are fulfilled:

(1) The sequence $v_{b+1} \in T v_{b}$ remains in $V$ and converge to $\eta$ with $Q$ - order $r$

(2) Now, we have to estimate for all $b \geq 0$ as

$$
d\left(v_{b}, \eta\right) \leq \mu^{s_{b}(r)} d\left(v_{0}, \eta\right)
$$

where $\mu=D\left(v_{0}\right)$ and $\omega$ is a nondecreasing nonnegative mapping on L satisfying $\varrho(e)=e \omega(e), \forall e \in L$

(3) $\forall$ we have to estimate $d\left(v_{b+1}, \eta\right) \leq \varphi\left(d\left(v_{b}, \eta\right)\right)$

Proof. As we know that $d(T v, \eta) \leq H(T v, T \eta)$, now substitute $v=\eta$ and obtain

$$
d(T \eta, \eta) \leq H(T \eta, T \eta) \leq \varrho(d(\eta, \eta))<d(\eta, \eta)=0,
$$

where $\eta \in T \eta$ and it is unique. Picard sequence converges with $Q$-order as

$$
\begin{aligned}
d\left(v_{b+1}, \eta\right) & \leq H\left(T_{v_{b}}, T \eta\right) \\
& \leq \varrho\left(d\left(v_{b}, \eta\right)\right) \\
& <d\left(v_{b}, \eta\right)=D\left(v_{b}\right) \\
& \leq C_{b} d\left(v_{b}, \eta\right)^{r}, \\
d\left(v_{b+1}, \eta\right) & \leq C_{b} d\left(v_{b}, \eta\right)^{r} .
\end{aligned}
$$


Now, we prove the other part by induction. Suppose $b=0$; then, equality holds $d\left(v_{0}, \eta\right)=d\left(v_{0}, \eta\right)$. Suppose that it is true for $b$,

$$
d\left(v_{b}, \eta\right) \leq \mu^{S_{b}(r)} d\left(v_{0}, \eta\right) .
$$

For $b+1$,

$$
\begin{aligned}
d\left(v_{b+1}, \eta\right) & \leq H\left(T_{v_{b}}, T \eta\right) \\
& \leq \varrho\left(d\left(v_{b}, \eta\right)\right) \\
& =d\left(v_{b}, \eta\right) \omega\left(d\left(v_{b}, \omega\right)\right) \\
& \leq \mu^{S_{b}(r)} d\left(v_{b}, \eta\right) \omega\left(D\left(v_{b}\right)\right) \\
& =\mu^{S_{b}(r)} d\left(v_{0}, \eta\right) \mu^{r^{b}} \\
& =\mu^{S b+1(r)} d\left(v_{0}, \eta\right) .
\end{aligned}
$$

Theorem 3. Assume a metric space $(V, d)$ and define a mapping $T: C \subset V \longrightarrow C B(V)$. The initial condition of $T$ is $D: C \longrightarrow \mathbb{R}_{+}$and gauge mapping $\varrho: L \longrightarrow L$ fulfills the condition $\varrho(e) \leq e$ for all $e \in L$. Let $\eta$ be in C. Assume that

$$
H(T v, \eta) \leq \gamma(D(v)) d(v, \eta), \quad \forall v \in C \text { with } D(\eta) \in L,
$$

where $\gamma$ is a nondecreasing mapping on $L$ such that e $\gamma(e)$ is a gauge mapping which is strict of order $r$ on $L$. Furthermore, for $e \in L: \omega(e)=0$, this implies that $\gamma(e)=0$ :

$$
\gamma(\varrho(e)) \leq \gamma(e)^{e}, \quad \forall e \in L .
$$

Now, for the fixed point of the mapping $T, \eta$ contains the set $E=\{v \in C: D(c) \in L\}$. Furthermore, for the starting point $v_{0}$ of $T$, the following statements hold:

(1) Picard sequence $v_{b+1} \in T_{v_{b}}$ remains in $V$ and converges to $\eta$ with order of convergence $R$-order $r$. It converges with Q-order $r$ given that $D(v) \leq B(v) d(v, \eta), \forall v \in C$, where $B: C \longrightarrow \mathbb{R}_{+}$is contentious at $\eta$.

(2) For each $b \geq 0$, we estimate that

$$
d\left(v_{b}, \eta\right) \leq g^{s_{b}(r)} d\left(v_{0}, \eta\right),
$$

where $g=\gamma\left(D\left(v_{0}\right)\right)$ and $\omega$ is nondecreasing and nonnegative on $L$ satisfying $\varrho(e)=r \omega(e)$.

Proof. First, we prove that $\eta$ is a fixed point which is unique:

$$
\begin{aligned}
d(T v, \eta) & \leq H(T v, T \eta), \\
H(T v, T \eta) & \leq d(v, \eta) .
\end{aligned}
$$

Now, substituting $v=\eta$ above, then

$$
\begin{aligned}
& d(T \eta, \eta) \leq H(T \eta, T \eta) \gamma(D(\eta)) d(\eta, \eta), \\
& d(T \eta, \eta) \leq 0 .
\end{aligned}
$$

This implies that $\eta \in T \eta$. Now, for uniqueness, suppose $\mu$ to be another fixed point and $\eta \neq \mu$, i.e.,

$$
d(\eta, \mu) \leq H(T \eta, T \mu) \leq \gamma(D(\eta)) d(\eta, \mu) \leq d(\eta, \mu),
$$

which implies that

$$
d(\eta, \mu)<d(\eta, \mu),
$$

which is a contradiction so $\eta=\mu$. Now, the first part of the theorem is already proved in the previous theorem. Now, we have the second part:

$$
\gamma\left(D\left(v_{b}\right)\right) \leq g^{(r)^{b}} .
$$

We prove it by mathematical induction. Take $b=0$; then, equality holds, i.e.,

$$
\gamma\left(D\left(v_{0}\right)\right)=\gamma\left(D\left(v_{0}\right)\right) .
$$

Assume that it is true for $n$. Now, we prove that for $b+1$,

$$
\begin{aligned}
\gamma\left(D\left(v_{b+1}\right)\right) & \leq \gamma\left(\varrho\left(v_{b}\right)\right) \\
& \leq\left[\gamma\left(D\left(v_{b}\right)\right)\right]^{r} \\
& \leq\left(g^{r^{b}}\right)^{r} \\
& =g^{r^{b+1}}, \\
H\left(T_{v_{b}}, T \eta\right) & \leq g^{r^{b}} d\left(v_{b}, \eta\right) .
\end{aligned}
$$

We prove this by mathematical induction; now, for $b=0$,

$$
d\left(v_{0}, \eta\right)=d\left(v_{0}, \eta\right)
$$

Assume that it is true for $b$; now, we prove that for $b+1$,

$$
d\left(v_{b+1}, \eta\right) \leq H\left(T_{v_{b}}, T \eta\right) \leq g^{r^{b}} d\left(v_{b}, \eta\right),
$$

and also note that

$$
d\left(v_{b}, \eta\right) \leq g^{s_{b}(r)} d\left(v_{0}, \eta\right) .
$$

Now,

$$
\begin{aligned}
d\left(v_{b+1}, \eta\right) & \leq H\left(T_{v_{b}}, T \eta\right) \\
& \leq g^{r^{b}+s_{b}(r)} d\left(v_{0}, \eta\right) \\
& =g^{r^{b+1}} d\left(v_{0}, \eta\right) .
\end{aligned}
$$

Hence,

$$
d\left(v_{b+1}, \eta\right) \leq g^{r^{b+1}} d\left(v_{0}, \eta\right)
$$

\section{Application}

We apply the proposed multivalued contraction mappings in integral theory.

Theorem 4. Consider the integral

$$
v(e) \in G \int_{e_{0}}^{e} p(\tau, v(\tau)) \mathrm{d} \tau+\eta, \quad \in G V_{v}(e)+\eta,
$$


where $V_{v}(e)=\int_{e_{0}}^{e} p(\tau, v(\tau)) d \tau, G$ is a compact subset of real line $\mathbb{R}$, and $p: \mathbb{R} \times \mathbb{R} \longrightarrow \mathbb{R}$ satisfies the following conditions:

(1) $p$ is continuous on the rectangle $R=\left\{(e, v):\left|e-e_{0}\right| \leq(1 / \lambda),|v-\eta| \leq\left(c / \lambda^{2}\right)\right\}, \quad$ where $\lambda=\max _{g \in G}|g|, 0<c<a$

(2) $p$ is continuous and bounded as $|p(e, v)|<\left(1 / \lambda^{2}\right)$ for all $(e, v) \in R$ :

$$
|p(e, v(e))-p(e, \eta(e))| \leq \gamma(y) \mid v(e)-\eta(e),
$$

where $y=|v(e)-\eta-(1 / \lambda)|$ with $v(e) \in L$ and $y \in I$. Then, the interval $I=\left[\left(e_{0}-1 / \lambda\right),\left(e_{0}+1 / \lambda\right)\right]$ has the solution of integral.

Proof. Let $C(I)$ denote those spaces which contain all those mappings which are continuous. And define metric space as $d(v, \eta)=\sup |v(e)-\eta(e)|$. Consider $\widetilde{C}$ to be a closed subspace of the space $C(I)$ which is defined as $\widetilde{C}:=\{v \in C(I): d(v, \eta) \leq(1 / \lambda)\}$. Define $T: \widetilde{C} \longrightarrow P(\widetilde{C})$ as

$$
T_{v}(t)=G \int_{e_{0}}^{e t} p(\tau, v(\tau)) \mathrm{d}(\tau)+\eta=G P_{v}(e)+\eta .
$$

Finding the solution of integral (56) becomes equivalent to the fixed point problem of (58). We represent that $T$ is well defined and $T$ is defined for every $v \in \widetilde{C}, T v$ is a compact subset of $\widetilde{C}$ for each $v \in \widetilde{C}$. For $s \in I$ and by definition of $\widetilde{C}$, we obtain $|v(s)-\eta| \leq(1 / \lambda)$. Thus, $(s, v(s)) \in R$. Since $p$ is continuous on $R$, way integral also exists. So $T$ is defined for each $v \in \widetilde{C}$. Now, we prove for each $v \in \widetilde{C}$ and $T v$ is a compact subset of $\widetilde{C}$. Let $f(e) \in T v(e)$. Then, $f(e)=g K_{v}(e)+\eta$ for some $g \in G$ and

$$
\begin{aligned}
|f(e)-\eta| & =\left|g P_{v}(e)\right| \\
& =\left|g \| P_{v} t(e)\right| \\
& \leq \lambda \int_{e_{0}}^{e}|P(\tau, v(\tau)) \mathrm{d} \tau| \\
& \leq \lambda \int_{e_{0}}^{e}|P(\tau, v(\tau))| \mathrm{d} \tau \\
& <\lambda \times\left(\frac{1}{\lambda^{2}}\right)=\frac{1}{\lambda},
\end{aligned}
$$

which prove that $d(f, \eta) \leq(1 / \lambda)$; then, $f \in \widetilde{C}$ since $f \in T v$ was arbitrary and so $T v \subset \widetilde{C}$ for each $v \in \widetilde{C}$. Now, for compactness, first we prove that $T x$ is compact. Consider a sequence $b_{n} \subset T v$ and $b_{n}=g_{n} P_{v}(t)+\eta$ for some $g_{n} \in G ; n=1,2,3, \ldots$. Since $G$ is compact, then there exists subsequence $\left\{g_{n_{i}}\right\}$ of $\left\{g_{n}\right\}$ such that $g_{n_{i}} \longrightarrow \tilde{g}$. Let $b=\widetilde{p} K_{v}(e)+\eta$; then, we obtain

$$
\begin{aligned}
d\left(b_{n}, b\right)= & \sup _{e \in I}\left(\mid g_{n_{i}}-\tilde{g} \| P_{v}(e)\right) \leq \mid g_{n_{i}} \\
& -\tilde{g}\left|\sup _{e \in I}\right| P_{v}(e) \mid \longrightarrow 0, \quad \text { as } j \longrightarrow \infty,
\end{aligned}
$$

and we note that $D(v)=d(v, T v)$, where $T_{v}(e)=G \int_{e_{0}}^{e} p(\tau, v(\tau)) \mathrm{d} \tau+\eta$ and

$$
\begin{aligned}
d(v, T v) & =\inf _{w \in T v} d(v, w) \\
& =\inf d\left(v, g \int_{e 0}^{e} p(\tau, v(\tau)) \mathrm{d} \tau+\eta\right) \\
& =\operatorname{infsup}\left|v(e)-g \int_{e 0}^{e} p(\tau, v(\tau)) \mathrm{d} \tau-\eta\right| \\
& =\operatorname{infsup}\left|v(e)-\eta-g \int e 0^{e} p(\tau, v(\tau)) \mathrm{d} \tau\right|,
\end{aligned}
$$

where inf apply on $g$ and $\lambda>\inf g$ :

$$
\begin{aligned}
& \geq \sup \left|v(e)-\eta-\lambda \int_{e 0}^{e} p(\tau, v(\tau)) \mathrm{d} \tau\right| \\
& \geq \sup \left|v(e)-\eta-\left(\frac{1}{\lambda}\right)\right| \\
& \geq \sup (w), \quad \forall w \in I \\
& =\sup \left|e_{0}+\left(\frac{1}{\lambda}\right)\right| \\
& \geq \sup (e), \quad \forall e \in I .
\end{aligned}
$$

Now, $\gamma$ is a nondecreasing function such that

$$
\gamma(D(v)) \geq \sup \gamma(w), \quad \forall w \in I .
$$

Furthermore, note that

$$
\begin{aligned}
H(T v, T \eta) & =H\left(G K_{v}(e)+\eta, G P_{\eta}(e)+\eta\right) \leq H\left(G P_{v}(e), G P_{\eta}(e)\right), \\
H\left(G P_{v}(e), G P_{\eta}(e)\right) & =\max \left\{\max _{a \in \mathrm{GP}_{v}}(e) d\left(a, G P_{\eta}(e)\right), \max _{b \in \mathrm{GP}_{\eta}} d\left(b, G P_{v}(e)\right)\right\} .
\end{aligned}
$$

Now, 


$$
\begin{aligned}
\max _{a \in \mathrm{GP}_{v}}(e) d\left(a, G P_{\eta}(e)\right) & =\max _{a \in \mathrm{GP}_{v}} \max _{b \in \mathrm{GP}_{\eta}} d(a, b) \\
& =\max _{g \in G} \max _{g^{*} \in G} d\left(g p(e, v), g^{*} p(e, \eta)\right) \\
& =\max g \in G \max _{g^{*} \in G} \sup _{e \in I}\left|g p(e, v)-g^{*} p(e, \eta)\right| \\
& \leq \max _{g \in G} \max _{g^{*} \in G} \sup _{e \in I}\left[\left|g p(e, \eta)-g^{*} p(e, \eta)\right|+|g p(e, \eta)-g p(e, v)|\right] \\
& \leq \max _{g \in G} \max _{g^{*} \in G}\left[|g| \sup _{e \in I}\left|g p(e, v), g^{*} p(e, \eta)\right|+\left|g-g^{*}\right| \sup _{e \in I}|p(e, \eta)|\right] \\
& =\max _{g} g \in G|g| \sup _{e \in I}|p(e, \eta)-p(e, v)| \\
& =\lambda \sup _{e \in I}|p(e, \eta)-p(e, v)| .
\end{aligned}
$$

and we have

$$
\begin{aligned}
|p(e, \eta)-p(e, v)| & \leq \int_{e 0}^{e}|p(\tau, \eta(\tau))-p(\tau, v(\tau))| \mathrm{d} \tau \\
& \leq \int_{e 0}^{e} \gamma(w) /|\eta(\tau)-v(\tau)| \mathrm{d} \tau \\
& \leq \sup _{e \in I} \gamma(w)|\eta(e)-v(e)| \int_{e 0}^{e} \mathrm{~d} \tau \\
& \leq \gamma(D(v)) d(v, \eta)\left|e-e_{0}\right| \\
& \leq \gamma(D(v)) \frac{1}{\lambda} d(v, \eta) .
\end{aligned}
$$

Thus, we obtain

$$
\begin{aligned}
\max a \in G P_{v} d\left(a, G P_{\eta}(e)\right) & \leq \lambda \gamma(D(v)) \frac{1}{\lambda} d(v, \eta) \\
& =\gamma(D(v)) d(v, \eta) .
\end{aligned}
$$

Now, interchanging $V$ and $\eta$, we obtain

$$
\max b \in G P_{\eta} d\left(b, G P_{v}(e)\right) \leq \gamma(D(\eta)) d(\eta, v) .
$$

Thus,

$$
H(T v, T \eta) \leq \gamma(D(v)) d(v, \eta)
$$

\section{Data Availability}

No data were used to support this study.

\section{Conflicts of Interest}

All authors declare that there are no conflicts of interest.

\section{Acknowledgments}

This research project received a grant from Anhui Jianzhu University. The authors acknowledge the technical and financial support.

\section{References}

[1] P. D. Proinov, "A generalization of the Banach contraction principle with high order of convergence of successive approximations," Nonlinear Analysis: Theory, Methods \& Applications, vol. 67, no. 8, pp. 2361-2369, 2007.

[2] Q. Kiran and T. Kamran, "Nadler's type principle with high order of convergence," Nonlinear Analysis: Theory, Methods \& Applications, vol. 69, no. 11, pp. 4106-4120, 2008.

[3] M. E. Gordji, H. Baghani, H. Khodaei, and M. Ramezani, "Geraghty's fixed point theorem for special multi-valued mappings," Thai Journal of Mathematics, vol. 10, no. 2, pp. 225-231, 2012.

[4] E. Kreyszig, Introductory Functional Analysis with Applications, Wiley, New York, NY, USA, 1978.

[5] S. Nadler Jr., "Multi-valued contraction mappings," Pacific Journal of Mathematics, vol. 30, no. 2, pp. 475-488, 1969.

[6] B. D. Rouhani and S. Moradi, "Common fixed point of multivalued generalized $\varphi$-weak contractive mappings," Fixed Point Theory and Applications, vol. 2010, p. 708984, 2010.

[7] Y. Song and H. Wang, "Convergence of iterative algorithms for multivalued mappings in Banach spaces," Nonlinear Analysis: Theory, Methods \& Applications, vol. 70, no. 4, pp. 1547-1556, 2009.

[8] V. Berinde, Iterative Approximation of Fixed Points, Springer, Berlin, Germany, 2007.

[9] F. E. Browder, "Multi-valued monotone nonlinear mappings and duality mappings in Banach spaces," Transactions of the American Mathematical Society, vol. 118, p. 338, 1965.

[10] P. Z. Daffer and H. Kaneko, "Fixed points of generalized contractive multi-valued mappings," Journal of Mathematical Analysis and Applications, vol. 192, no. 2, pp. 655-666, 1995.

[11] B. Fisher, "Common fixed-point theorems for mappings and set-valued mappings," Journal of the University of Kuwait (Science), vol. 11, no. 1, pp. 15-22, 1984.

[12] S. Itoh and W. Takahashi, "Single-valued mappings, multivalued mappings and fixed-point theorems," Journal of Mathematical Analysis and Applications, vol. 59, no. 3, pp. 514-521, 1977.

[13] A. Jourani and L. Thibault, "Verifiable conditions for openness and regularity of multivalued mappings in Banach spaces," Transactions of the American Mathematical Society, vol. 347, no. 4, pp. 1255-1268, 1995.

[14] T. Kamran, M. Samreen, and Q. UL Ain, "A generalization of b-metric space and some fixed point theorems," Mathematics, vol. 5, no. 2 , p. $19,2017$. 
[15] N. Mizoguchi and W. Takahashi, "Fixed point theorems for multivalued mappings on complete metric spaces," Journal of Mathematical Analysis and Applications, vol. 141, no. 1, pp. 177-188, 1989.

[16] A. Petrusel, "Local fixed point results for graphic contractions," Journal of Nonlinear Variance Analysis, pp. 141-148. In press.

[17] T. M. M. Sow, "Strong convergence of a modified Mann algorithm for multivalued quasi-nonexpansive mappings and monotone mappings with an application," Communications in Optimization Theory, vol. 2020, pp. 1-14, Article ID 3, 2020.

[18] A. Petrusel, "Gabriela Petrusel, Fixed point results for multivalued locally contractive operators," Appl. Set-Valued Anal. Optim, vol. 2, pp. 175-181, 2020.

[19] P. D. Proinov, "General local convergence theory for a class of iterative processes and its applications to Newton's method," Journal of Complexity, vol. 25, no. 1, pp. 38-62, 2009. 